Stefan Kleiner (Mannheim)

\title{
Die Neuauflage des Duden-Aussprache- wörterbuchs - Allgemeine Neuerungen und Umsetzung empirischer Erkenntnisse aus dem Korpus „Deutsch heute“
}

\begin{abstract}
Im Jahr 2015 ist die 7. Auflage des Duden-Aussprachewörterbuchs erschienen, für deren Bearbeitung erstmals die MitarbeiterInnen des IDS-Projekts „Gesprochenes Deutsch“ verantwortlich zeichneten. Im vorliegenden Beitrag werden die konzeptionellen und inhaltlichen Veränderungen beschrieben, die in der Neuauflage umgesetzt wurden. Sie lassen sich im Wesentlichen unter dem Motto „Hinwendung zur Deskriptivität“ zusammenfassen. Neben den üblichen lexikografischen Prozeduren wie der Streichung veralteter Lemmata und der Erweiterung des Lemmabestands um bisher nicht dokumentierte Wörter sind zunächst im Einleitungsteil Kapitel ergänzt, vollständig überarbeitet oder völlig neu erstellt worden. Systematische Veränderungen wurden bei verschiedenen Transkriptionskonventionen vorgenommen (z.B. bei der Notation der Diphthonge). Die wesentlichste Neuerung ist jedoch die Einbeziehung von empirischen Daten zum deutschen Gebrauchsstandard vor allem aus dem Projektkorpus „Deutsch heute“, die es ermöglicht haben, fundierte Angaben zur regionalen Verbreitung von Aussprachevarianten zu machen.
\end{abstract}

\section{Einleitung}

In einem Vortrag auf der IDS-Jahrestagung 2013 (Kleiner 2014) hatte sich der Autor mit den bis dato bestehenden deutschen Aussprachekodizes auseinandergesetzt und eine Reihe der in ihnen verzeichneten Formen auf den empirischen Prüfstand gestellt. Zu diesem Zweck wurde zunächst die Gebräuchlichkeit der betreffenden Formen in dem von 2006 bis 2009 im ganzen deutschen Sprachraum hauptsächlich mit Oberstufenschülern an Gymnasien erhobenen Korpus „Deutsch heute“ (Korpusbeschreibungen z.B. bei Brinkmann et al. 2008; Kleiner 2015) ermittelt. Für diesen, häufig regional variierenden, Gebrauchsstandard konnten hinsichtlich der thematisierten Phänomene meist deutliche Abweichungen von den Forderungen der Aussprachewörterbücher ermittelt werden. Als Fazit wurde in der Quintessenz eine stärker deskriptive Ausrichtung der Kodizes gefordert, wie sie für das Englische vorbildhaft im „Longman pronunciation dictionary“ 
(Wells 1990, 2008) umgesetzt ist. Abschließend wurde darüber spekuliert, ob bei einer möglichen Neuauflage des Aussprachewörterbuchs des Dudenverlags (Mangold 2005) „konzeptionelle Veränderungen im Sinne der hier präsentierten Vorschläge vorgenommen werden“ (Kleiner 2014, S. 295).

Bereits bald darauf, im Juni 2014, erreichte unsere Projektgruppe eine Anfrage der Dudenredaktion, in der uns die Autorschaft einer Neuauflage von Dudenband 6, d.h. dem Aussprachewörterbuch, angetragen wurde. Dass wir so schnell tatsächlich in die Lage versetzt würden, die gerade erst in Kleiner (2014) schriftlich ausformulierten programmatischen Vorschläge und Forderungen eigenhändig in die Praxis umzusetzen, war nicht abzusehen gewesen. Allerdings waren die zeitlichen Rahmenbedingungen alles andere als günstig, denn der Erscheinungstermin für die dann 7. Auflage war bereits unverrückbar auf Herbst 2015 festgelegt, was letztlich bedeutete, dass uns für die Überarbeitung alles in allem nur neun Monate effektiver Arbeitszeit (von Oktober 2014 bis Juni 2015) zur Verfügung standen. Es war darum nötig, neben zwei durch Verlagsmittel finanzierten zusätzlichen Hilfskraftstellen auch die drei bereits im Projekt tätigen Hilfskräfte vollumfänglich in die Arbeiten einzubinden, sodass zusammen mit den beiden wissenschaftlichen Projektmitarbeitern Ralf Knöbl und Stefan Kleiner insgesamt sieben Personen hauptamtlich an der Neuauflage mitgewirkt haben. ${ }^{1}$

\section{Zum Standard-Begriff der Neuauflage}

Anders als auf der schriftlichen Ebene, wo für die Orthografie eine amtliche Regelung besteht, gibt es für die mündliche Realisierung der deutschen Standardsprache keine offiziellen Regelungen, die bestimmte Ausspracheweisen verbindlich vorschreiben. Das macht einerseits den oft verwendeten, parallel zu Orthografie gebildeten Terminus Orthoepie per se problematisch, denn es fehlt damit auf der mündlichen Ebene der offiziell legitimierte Bezugspunkt für die Aufstellung von Regeln, mit dem sich die Bezeichnung Orthoepie („Rechtlautung“) rechtfertigen ließe. Andererseits eröffnen sich für Kodifikatoren verschiedene Möglichkeiten, diesen Bezugspunkt selbst zu setzen. Die ursprüngliche Kodifikation der SiebsKommission (Siebs (Hg.) 1898) orientierte sich an der Sprache der Bühne, sie war zunächst auch primär als vereinheitlichende Regelung für die Bühnenaussprache

1 Hilfskräfte waren Judith Linneweber, Alexandra Lorson, Madlen Nimz, Pia Rübig und Hristo Velkov. Zusätzlich wurde das Projekt von Verlagsseite von Franziska Münzberg und Carsten Pellengahr durch redaktionelle Tätigkeiten sowie von Thorsten Frank durch informatische Expertise unterstützt. 
gedacht. Deren Skopus wurde aber bald zu einer allgemeinen Aussprachenorm für das Deutsche erweitert. Mit dem „Wörterbuch der deutschen Aussprache“ (Krech et al. (Hg.) 1964) wurde dann der Sprachgebrauch von Nachrichtensprechern, ebenfalls eine sprachlich speziell geschulte Berufsgruppe, zur Richtschnur für die korrekte Aussprache der Gesamtbevölkerung gemacht. In dieser Tradition steht auch das 2009 erschienene „Deutsche Aussprachewörterbuch“ (Krech et al. 2009). Nicht zuletzt haben sich die Vorgängerauflagen des Duden-Aussprachewörterbuchs seit der 2. Auflage (Mangold 1974) explizit an den Ergebnissen von Krech et al. (Hg.) (1964) orientiert (vgl. Mangold 2005, S. 34).

Für die von unserem IDS-Projekt bearbeitete Neuauflage haben wir hingegen versucht, dem Bezugspunkt „Berufssprecher“ und der dadurch bedingten dominant präskriptiven Sichtweise durch die Einbeziehung des Gebrauchsstandards, d.h. von in formeller Situation (z.B. beim Vorlesen) produzierten Aussprachevarianten durch nicht in Sprechberufen tätige Laien, eine stärker deskriptive Ausrichtung zu geben. Wir vertreten, anders als bisherige Aussprachewörterbücher des Deutschen, mithin die Auffassung, dass es Aufgabe eines Aussprachewörterbuchs ist, gebrauchshäufige Varianten nicht auszublenden, sondern, im Gegenteil, ausdrücklich zu dokumentieren. Die betreffenden Varianten werden, das zeigen empirische Daten, zum Teil von Millionen deutscher MuttersprachlerInnen verwendet und sind damit Formen ihres Gebrauchsstandards. Sie wurden in früheren Aussprachewörterbüchern häufig genau deshalb nicht verzeichnet, weil es sich um nur in Teilen des deutschen Sprachraums gebräuchliche Formen handelt. Die z.B. von Schmidt/Herrgen (2011) vertretene Auffassung, dass Standard(aus)sprache nur dann vorliege, solange keine sprachlichen Regionalismen verwendet werden, teilen wir demnach nicht.

\section{Die Neubearbeitung}

Der eigentlichen Überarbeitung ging eine Phase voraus, in der die vorhergehende 6. Auflage (Mangold 2005) genauer unter die Lupe genommen wurde. Recht bald stellte sich bei dieser Sichtung, dann auch im Vergleich mit vorhergehenden Auflagen bis zur Erstauflage (Mangold 1962) heraus, dass einerseits die langjährige Autorschaft von Max Mangold dem Werk eine spezifische Ausrichtung gegeben hatte, die nur mit erheblichem Aufwand in die von uns gewünschte Richtung würde verändert werden können, und dass andererseits der Stand des Wörterbuchs - abgesehen von Wortschatzerweiterungen und geringen Modifikationen im Einleitungsteil - im Kern noch dem der 2. Auflage von 1974 entsprach. 


\subsection{Ziel der Neubearbeitung: Hinwendung zur Deskriptivität}

Das in Kleiner (2014) als Hinwendung zur Deskriptivität formulierte Programm sollte besonders durch die folgenden Maßnahmen in der 7. Auflage des DudenAussprachewörterbuchs verwirklicht werden:

- Berücksichtigung der Standardaussprache auch von Nicht-BerufssprecherInnen („Laien“) unter der Bedingung, dass diese über eine höhere (d.h. gymnasiale) Schulbildung verfügen, die sowohl passive Kenntnis als auch Übung im aktiven Gebrauch der Standardsprache („Gebrauchsstandard“) gewährleisten soll; ${ }^{2}$

- Berücksichtigung von gebräuchlichen Aussprachevarianten, auch wenn sie von den regulären Graphem-Phonem-Beziehungen des Deutschen oder von der traditionellen Kodifikation abweichen (z.B. [i] in ging, Viertel, [ks] in nächst).

- Auszeichnung von nationalen und großregionalen Provenienzen von Aussprachevarianten, sofern diese aufgrund von empirischen Daten oder Expertenwissen bekannt sind;

- Ermittlung von Präferenzen für Aussprachevarianten ausgewählter Wörter mittels einer Umfrage in der Bevölkerung.

Die „Programmpunkte“ sind in wesentlichen Teilen vom „Longman Pronunciation Dictionary“ inspiriert, das diese bereits in seiner ersten Auflage (Wells 1990) umgesetzt hat.

\subsection{Veränderungen im Einleitungsteil}

Bei unserer Überarbeitung haben wir zwar die grundsätzliche Struktur des Einleitungsteils beibehalten, aber inhaltlich doch einerseits erhebliche Veränderungen, andererseits umfangreiche Erweiterungen vorgenommen. Die Folge war, dass dessen Umfang von 119 Seiten in der 6. auf 151 Seiten in der 7. Auflage angestiegen ist. Hauptverantwortlich für den Seitenzuwachs war die Überarbeitung des Kapitels „Deutsche Aussprachelehre“, in dem die Graphem-Phonem-Korrespondenzen

2 Damit folgen wir dem v.a. in der anglistischen Linguistik (und damit auch der dortigen Kodifikationspraxis) gängigen Konzept, das davon ausgeht, dass für die Beschreibung der Standard(aus)sprache die Beobachtung des tatsächlichen Sprachgebrauchs der „educated speakers“ maßgeblich sei (vgl. z.B. Wells 2008, S. XVII, XIX). Auch Werner König erhebt für seinen Ausspracheatlas nur Daten von Studierenden, wobei zusätzlich mindestens ein Elternteil Abitur haben musste (vgl. König 1989, Bd. 1, S. 17). 
des Deutschen (inklusive der in englischen, französischen, spanischen und italienischen Lehnwörtern gebräuchlichen) detailliert beschrieben werden. Hier haben wir eine systematische Neuordnung vorgenommen und die vormals nach graphotaktischen Kriterien durchgeführte Sortierung in eine phonotaktische überführt.

Weitere Veränderungen wurden an verschiedenen Stellen des Einleitungsteils umgesetzt. Die wichtigsten davon sind:

- Ergänzung der bereits vorhandenen Ausspracheangaben für Fremdsprachen um in der 6. Auflage nicht enthaltene Tabellen zu Bulgarisch, Englisch, Französisch, Russisch und Ukrainisch;

- Abschaffung des Konzepts von „genormter“ und „ungenormter“ Lautung und Ersatz der betreffenden Abschnitte durch Kapitel zur „Standardaussprache des Deutschen“ und „Variation in der Standardaussprache“;

- Streichung des - ohnehin recht kurzen - Abschnitts zur „Bühnenaussprache“;

- neue Kapitel über „Schwache Wortformen“ (von Artikeln, Pronomen, Präpositionen) und „Zur Aussprache von Entlehnungen aus dem Englischen“, weil sich durch die Dominanz des Englischen als Weltsprache und die damit verbundene hohe Frequenz englischer Entlehnungen im Deutschen bei diesen ein breites und von Phänomen zu Phänomen recht unterschiedliches Oszillationsspektrum zwischen der britischen/amerikanischen Originalaussprache und mehr oder weniger eingedeutschten Varianten ergibt.

\subsection{Streichungen}

Im Duden-Aussprachewörterbuch wurde zwar wie üblich bei Neuauflagen der Wortschatz erweitert, einmal Gebuchtes blieb danach aber anscheinend unangetastet. Entsprechend hatte sich über die Jahre ein Bestand an obsoletem Wortschatz - auch im onomastischen Bereich ${ }^{3}$ - angesammelt. Eine ganze Reihe veralteter oder in dieser Form heute ungebräuchlicher Lexeme wie mosleminisch, anhägern oder bankerott und im Namenbereich heute nur mehr historische Namen wie Stalinstadt (schon seit 1961 Eisenhüttenstadt), Panamerican Airways, Obervolta, Comecon wurden darum von uns gestrichen. Ebenfalls getilgt wurde eine große Zahl an heute unüblichen orthografischen Varianten wie Aszese, Kapriccio, Karrag[h]een, Kanossa, Tschingis Chan, Wagadugu, Catchup, Shampoon, bordorot.

3 Wie in allen Aussprachewörterbüchern besteht auch im Dudenband 6 ein wesentlicher Teil der gebuchten Lemmata aus Orts- und Personennamen (grob hochgerechnet sind ungefähr ein Drittel der Wortliste Namen). 
Eine Besonderheit der Vorgängerauflagen war, dass zu Verben, deren Stamm auf Leniskonsonanten endet oder um -ig-, -el-, -er- erweitert ist, systematisch bestimmte Flexionsformen verzeichnet waren: endungslose Imperative des Typs egg!, grab!, mobb!, nächtig!, bewald!, Formen der 3. Pers. Sg. wie in sagt, schreibt, schädigt und die 1. Pers. Sg./Imperativ wie in nagle, gable, ordre. Grund dafür, diese Flexionsformen explizit aufzuführen, war in den ersten beiden Fällen sicherlich die eintretende Fortisierung zu [p, t, k] bzw. bei -ig- die Spirantisierung zu [ç] im Auslaut bzw. vor /t/, im letzteren Fall vermutlich, dass hier, trotz eines nachfolgenden Konsonanten, gerade keine Fortisierung eintritt. Da diese Prozesse einerseits völlig regelhaft und damit die lautlichen Ergebnisse vorhersagbar sind, andererseits gerade unter den endungslosen Imperativen nicht wenige zwar potenziell bildbare, aber ungebräuchliche Wortformen enthalten waren (bewald!, beselig!, sänftig!), wurden all diese Einträge gestrichen. Insgesamt wurden ca. 1.650 Streichungen vorgenommen.

\subsection{Neuaufnahmen}

Der üblichste Vorgang bei Neuauflagen von Wörterbüchern ist sicherlich die Erweiterung durch die Aufnahme neuer, in der Regel seit der vorhergehenden Auflage in Gebrauch gekommener Lexeme. In der 7. Auflage des Duden-Aussprachewörterbuchs wurden insgesamt ca. 3.100 neue Lemmata aufgenommen. Um mögliche Aufnahmekandidaten zu ermitteln, wurden verschiedene Quellen genutzt: eine aus dem Deutschen Referenzkorpus ermittelte Wortfrequenzliste (DeReWo 2013 ${ }^{4}$ ) sowie die Neuaufnahmelisten der Dudenbände 1 (Orthografie) und 5 (Fremdwörter). ${ }^{5}$ Daneben wurden eigene Recherchen angestellt und fokussiert auf bestimmte semantische Bereiche, in denen mit einer größeren Zahl an Neuentlehnungen zu rechnen war (u.a. Nahrungsmittel, z.B. Aceto balsamico, Börek, Chia-Samen, Goji-Beere, Guarkernmehl, Lahmacun, Quinoa, Sencha, oder Termini aus dem IT-Bereich wie Excel, Firewall, QR-Code, Tablet, Thread). Außerdem wurde eine gewisse Zahl hochfrequenter Komposita des Deutschen wie Flughafen, Kindergarten, Staatsanwaltschaft integriert. ${ }^{6}$ Im Namenbereich wurden besonders die

\footnotetext{
4 Internet: www1.ids-mannheim.de/kl/projekte/methoden/derewo.html (Stand: 28.9.2018).

5 Dank an Rainer Perkuhn für seine Unterstützung bei der Aufbereitung dieser Wortlisten!

6 Komposita aus nativ deutschen Bestandteilen sind im Duden-Aussprachewörterbuch traditionell nur in geringer Zahl enthalten. Grund dafür ist vermutlich, dass sich die Aussprache der Kompositionsglieder im Kompositum nicht von den entsprechenden Simplizia unterscheidet und auch der Wortakzent bei den die Masse der Kompositionen stellenden zweigliedrigen Determinativkomposita regelmäßig auf dem Bestimmungswort liegt.
} 
damals aktuellen nationalen und internationalen Politiker neu aufgenommen wobei Namen von mittlerweile aus ihren Ämtern Ausgeschiedenen wie Hollande oder Jazenjuk zeigen, dass Aktualität in diesem Bereich schon nach relativ kurzer Zeit nicht mehr gegeben ist.

\subsection{Veränderungen bei Transkriptionen}

Die lautschriftlichen Transkriptionen im Wörterbuchteil wurden in verschiedener Hinsicht überarbeitet. Einerseits handelt es sich dabei lediglich um Anpassungen bei bestimmten formellen Konventionen - z.B. bei der Verwendung von Diakritika zur Markierung von Akzentsitz oder von Diphthongen -, die von einer dudenspezifischen auf eine allgemeingültige Notation umgestellt wurden. Andererseits wurde vor allem versucht, die aus unseren empirischen Daten gewonnenen Informationen dazu zu nutzen, die Transkriptionen realitätsnäher zu machen. Im Folgenden sind diese systematischen Veränderungen listenartig aufgezählt:

1. In den Wörterbüchern des Dudenverlags sind die orthografischen Formen der Lemmata traditionell mit einem aus untergesetzten Punkten und Strichen bestehenden diakritischen System angereichert, an dem der Wortakzent und die Quantität des akzentuierten Vokals abgelesen werden können: Bei „sẹngen“ ist der erste Vokal akzentuiert und kurz, bei „Semem“ ist der zweite akzentuiert und lang. Da es gerade zum Wesen eines Aussprachewörterbuchs gehört, bei jedem Lemma eine detaillierte Ausspracheangabe in Lautschrift zu verzeichnen, konnte auf diese - in anderen Wörterbuchtypen sinnvolle - Angabe ohne Informationsverlust verzichtet werden. ${ }^{7}$

2. Bei den lautschriftlichen Notationen für Affrikaten und Diphthonge wurde das bisher verwendete Diakritikum, der untergesetzte, zwei Zeichen umspannende Bogen, entweder gestrichen (Affrikaten: [pf, ts, t $\left[\right.$, dz] > [pf, ts, t $\left.\int, d z\right]$ ) oder durch das IPA-Diakritikum ,unsilbisch“ unter dem Diphthongzweitglied ersetzt ([ai, au, כy] > [ar, av, , ગn] $]$ ).

3. Für die Diphthongzweitglieder werden neu, analog zur Darstellung z.B. in Kohler (1995), die Zeichen für die zentralisierten hohen Vokale verwendet, weil dies die maximale Höhe ist, die der Glide der Diphthonge in der überregionalen Standardaussprache des Deutschen in der Regel erreicht. ${ }^{8}$

7 Gegen die Beibehaltung der Diakritika sprach auch der erhebliche zusätzliche Arbeitsaufwand, die deren Pflege im von uns zur Überarbeitung verwendeten Redaktionssystem verursacht hätte. 8 Eine Notation [aen, ao, , əe] mit gespannten halbhohen Zweitgliedern wäre allerdings lautlich noch realistischer gewesen. 
4. Der <eu>, <äu> (v.a. in süddeutschen Namen auch <oi>) geschriebene Diphthong wird neu mit ungerundetem Zweitglied notiert: [əy] > [ગ્n]. Daten aus dem Korpus „Deutsch heute“ (aber auch von BerufssprecherInnen) zeigen eindeutig, was schon vor hundert Jahren von Otto Bremer (1918, S. 34) völlig zutreffend festgestellt wurde: Das Zweitglied ist - abgesehen von koartikulatorischen Einflüssen durch folgende rundende Konsonanten und vom Usus in bestimmten Sprachlandschaften - im Deutschen in aller Regel ungerundet.

5. Zwischen einer wortinternen Abfolge von unbetontem und betontem Vokal ohne morphologische Grenze oder vor einem Suffix wurde in den Transkriptionen konsequent ein optionaler (darum in eckige Klammern gesetzter) Glottalverschluss eingeführt („ru[l]'i:nə“, „aktu'[l]cl“, „te'[l]a:te“). Im Korpus „Deutsch heute“ ist die Erscheinung vor allem in Mittel- und Norddeutschland belegt (vgl. Kleiner 2014, S. 283-285), seltener in Süddeutschland und praktisch nie in Österreich und der Schweiz.

6. Die im Deutschen übliche progressive Stimmlosigkeitsassimilation, d.h., dass inhärent stimmhafte Konsonanten nach einem vorhergehenden stimmlosen Konsonanten ihre Stimmlosigkeit verlieren, wurde im Fall von [z] als [z] in die Transkriptionen übernommen („,beabsichtigen bə'|apżçtıgn““9). Bei [b, d, g, $\mathrm{v}, 3]$ wurde hingegen keine entsprechende Markierung vorgenommen, obwohl die Assimilation auch bei diesen Leniskonsonanten auftritt. Grund für diese unterschiedliche Behandlung war, dass die phonetische Auffälligkeit von [z] vs. [z] am höchsten ist, wohingegen sie besonders bei den Plosiven wenig auffällt, besonders weil entstimmte [b, d, g] auch im absoluten Anlaut im Deutschen insgesamt sogar üblicher sind als voll stimmhafte Realisierungen.

7. Zahlreiche Veränderungen wurden bei der Klassifikation von vormals als [i], [u] und [ $\breve{y}]$, d.h. als unsilbisch markierten hohen Vokalen durchgeführt, die in der Neuauflage silbisch als [i], [u] und [y] wiedergegeben werden: „Piano pi'a:no“,Klavier“ (vormals [pi'a:no]) vs. „piano pi'a:no“ ,leise“, „Viola vi'o:la“ (vorm. [vi'o:la]), „Linguistik lingu'[l]Istrk“ (neben, wie vorm. nur, [lı' gu्nistrk]), „Ruanda ru'[l]anda“ (vorm. [rứanda]), „Cyanat tsya'na:t“ (vorm. [tsy̆a'na:t]).

8. Neu eingeführt wurde das IPA-Zeichen zur Markierung eines Nebenakzents bei mindestens dreisilbigen Wörtern („Altkatholik 'altkatoli:k“ (vorm. ['altkato'li:k])), da gleichrangige Hauptakzente im selben Lexem im Deutschen außerhalb von Topikalisierungen unüblich sind. Im Zuge dieser Änderungen wurden auch bei allen Transkriptionen mit bisher zwei (oder mehr)

9 Zitate konkreter Lemmata aus der Neuauflage werden typografisch wie im Original wiedergegeben, d.h. mit Fettdruck des Stichworts und ohne Auszeichnung der Lautschrift durch phonetische Klammern. 
Hauptakzenten in einem Wort alle bis auf einen Hauptakzent gestrichen (,deshalb 'deshalp“ (vorm. ['des'halp])).

9. Veränderungen bei Transkriptionen von englischen Lehnwörtern:

a) Für die vormals nur vollständig eingedeutscht als Monophthonge [e:] und [o:] notierten Entsprechungen von engl. [eI] und [əত্র] wurde bei jedem betreffenden Lexem in Einzelfallentscheidungen anhand von Korpusdaten oder Expertenmeinung entschieden, ob Monophthong- oder Diphthongaussprache im Deutschen heute üblicher ist, und danach die betreffenden Varianten gereiht bzw. durch Quantifikatoren ein Anhaltspunkt zu deren Häufigkeit gegeben: „Container kon'te:ne, kon'tعıne“; „Update 'apde्राt, auch:' 'apde:t“; „Baby 'be:bi, 'beribi“, „Babyboom[er] 'beribibu:m[e], auch: ' be:bibu:m[e]“; „Bowle 'bo:lə“; „Oldie 'o:ldi, 'Jv్ldi“; „Holding

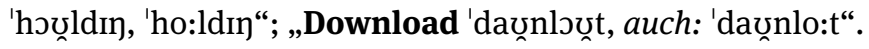

b) Bisher in Wörtern wie Call-by-Call, Launch, Squaw, Wall Street verwendetes [o:] wurde systematisch durch der tatsächlichen Aussprache im Deutschen näherstehendes [ə:] ersetzt (,ko:lbar'ko:1“ usw.).

c) Die bisher für den Vokal in Entlehnungen wie Band, Fan, Gag, Gang ausschließlich verwendete vollständig eingedeutschte Transkription $[\varepsilon]$ wurde zu [ $\varepsilon[:]]$ erweitert, um anzuzeigen, dass der Vokal im tatsächlichen Sprachgebrauch häufig (wie engl. /æ/) gedehnt gesprochen wird ([bent,

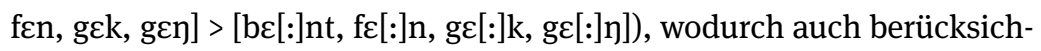
tigt wird, dass Gag und Geck für die meisten deutschen MuttersprachlerInnen nicht homophon sind.

d) Wortinitiales $<$ s $>$ in englischen Lehnwörtern wie Sex, Single, Song wurde bisher eingedeutscht als [z] wiedergegeben. Die tatsächliche Realisierung ist aber, auch in Mittel- und Norddeutschland, in aller Regel stimmloses [s] wie im Englischen (sodass sechs [zcks] und Sex [scks] ein Minimalpaar bilden); darum wurde in solchen Fällen [z] durch [s] ersetzt.

e) Trotz gewisser Bedenken wird die eingedeutschte Transkription [v] für englisch $\langle\mathrm{w}\rangle-/ \mathrm{w} /$ (World Wide Web [vœ:əحlt vart 'vep]) weiterhin als einzige Variante verzeichnet, obwohl klar ist, dass eine am Englischen orientierte Realisierung mit [w] heute im Deutschen weit verbreitet ist. Grund für die Nichtaufnahme war, „dass verlässliche empirische Daten zum tatsächlichen Gebrauch fehlen“ (Kleiner/Knöbl/Mangold 2015, S. 78).

10. In den über 9.000 gebuchten Namen englischen Ursprungs wurden verschiedene Anpassungen der Transkriptionen gemäß den Vorgaben von Wells (2008) vorgenommen. Besonders aufwendig war die Ergänzung der bisher vollständig fehlenden Diakritika sowie die Einführung von Regularitäten zur Variation zwischen silbischen Konsonanten und Schwa (,Snowdon 'snoư⿰亻”“ (vorm. [snoudn]) vs. „Stetson 'stetsən“ (vorm. [stetsn])). 
11. Bei französischen Lehnwörtern wurden systematische Anpassungen bei den Transkriptionen der nasalierten Vokale vorgenommen: Die in der Vorgängerauflage verwendeten [ã], [õ] und [õ̃] wurden ersetzt durch der tatsächlichen

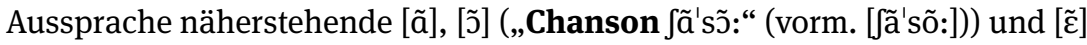
(„Parfum par'fy:m, auch: par'f̃̃ :“ (vorm. nur [par'foẽ:])).

\subsection{Kennzeichnung nationaler und großregionaler subnationaler Varianten}

Die aus unserer Sicht wesentlichste Neuerung der 7. Auflage ist die Einführung einer Kennzeichnung der regionalen Verbreitung von Aussprachevarianten. Eine Markierung von österreichischen und Deutschschweizer Varianten gab es zwar bereits in der letzten Auflage des Siebs (1969), und im Österreichischen Aussprachewörterbuch (Muhr 2008) wurden sogar für 12.000 Stichwörter konsequent trinationale Ausspracheangaben gemacht, ${ }^{10}$ aber in keinem der beiden Fälle wurde versucht, auch großregionale Variation (z.B. süd-, mittel-, norddeutsch oder ggf. noch genauer südost- oder südwestdeutsch) innerhalb der Nationen (d.h. insbesondere innerhalb Deutschlands) darzustellen. Aus Platz- und Übersichtlichkeitsgründen wurde im Wörterverzeichnis nur die Variation in Deutschland, Österreich und der Schweiz berücksichtigt und dokumentiert, auf die entsprechenden Verhältnisse in Südtirol, Luxemburg und Ostbelgien wird hingegen an bestimmten Stellen im Einleitungsteil eingegangen.

Die Gesamtmenge der im Duden-Aussprachewörterbuch neu als national/ regional markierten Lemmata umfasst mit insgesamt ca. 500 Stichwörtern zwar weniger als ein halbes Prozent des Gesamtwortschatzes, darunter befinden sich aber zahlreiche höherfrequente Lexeme und vor allem auch solche, die zu den allgemein bekannten lautlichen Variationsfällen im deutschen Sprachraum zählen. Dabei konzentriert sich die auf diese Weise markierte Variation auf bestimmte lautliche Bereiche:

1. Quantitäts- bzw. Gespanntheitsvariation, d.h. die Variation zwischen langen/ kurzen bzw. gespannten/ungespannten Vokalen: z.B. /i:/ - /I/ in Viertel, /ø:/ - /œ/ in rösten, /a/ - /a:/ in Rad, Bad

10 Die an sich lobenswerte Konzeption des Österreichischen Aussprachewörterbuchs wird noch durch eine wertvolle Tondatenbank bereichert, in der sich alle trinationalen Tonbelege (von je zwei BerufssprecherInnen pro Nation eingesprochen) anhören lassen. Leider kann das Werk vor allem aufgrund zahlreicher Fehler in den Transkriptionen seinen Anspruch als Kodex kaum erfüllen (dazu genauer Kleiner 2008; Wiesinger 2009a). 
2. Fremdwortphonologie, z.B. Variation bei ausgangssprachlich nasalierten Vokalen in Wörtern französischer Herkunft (Ballon, Balance), Aussprache von $<\mathrm{ch}>$ im Anlaut von Wörtern wie Chemie, Chirurg, Chile, Chrom, Aussprache von $<\mathrm{j}>$ im Anlaut in Jury, Journalist usw.

3. Wortakzentvariation wie z.B. in Ammoniak, Balkon, Mathematik.

Tatsächlich war ein erheblicher Teil der jetzt in der 7. Auflage neu regional markierten Aussprachevarianten an sich bereits in der 6. Auflage verzeichnet. Das Duden-Aussprachewörterbuch war damit auch schon in früheren Auflagen variantenfreundlicher als Siebs (1969) oder Krech et al. (2009). ${ }^{11}$

Das Wissen um die Diatopik der Varianten stammt im Wesentlichen aus drei verschiedenen Quellen. In der Literatur finden sich wertvolle Angaben vor allem in den entsprechenden Kapiteln zur „Standardaussprache in Österreich“(Wiesinger 2009b) und zur „Standardaussprache in der deutschen Schweiz“ (Haas/Hove 2009) des „Deutschen Aussprachewörterbuchs“ (Krech et al. 2009), außerdem vereinzelt im „Variantenwörterbuch der deutschen Standardsprache“ (Ammon et al. 2004) und im „Atlas zur deutschen Alltagssprache“ (Elspaß/Möller 2003 ff.).

Die zweite Informationsquelle zu regionalen Varianten der Standardaussprache waren „Gewährspersonen“ (in der Regel SprachwissenschaftlerInnen) aus verschiedenen Gegenden des deutschen Sprachraums. Sie kontaktierten wir einerseits in der Regel per E-Mail und fragten nach der Üblichkeit bestimmter Aussprachevarianten in den jeweiligen Regionen, andererseits nutzten wir auch das am IDS versammelte Potenzial an MitarbeiterInnen aus dem ganzen deutschen Sprachraum, indem wir mit den an Aussprache Interessierten in mehreren workshopartigen Sitzungen insgesamt einige hundert Varianten diskutierten. (Nicht für alle solchermaßen diskutierten Formen war dann letztlich ein regional begrenztes Vorkommen zu ermitteln, aber häufig konnten auf diese Weise klare Präferenzen ermittelt werden, was dann zu Erweiterungen bzw. Umformulierungen der betreffenden Wortartikel geführt hat.)

Die dritte und hinsichtlich regionaler Aussprachevariation detaillierteste Informationsquelle war schließlich unser Korpus „Deutsch heute“. Von den darin enthaltenen Aufnahmen von insgesamt 840 SprecherInnen aus dem ganzen deutschen Sprachraum wurde zu diesem Zweck vor allem die Vorleseaussprache der insgesamt gut 1.000 lexikalische Einheiten enthaltenden Wortliste herangezogen. Im Folgenden soll an den Beispielen rösten und Ballon/Balkon gezeigt werden, in welcher Weise die Empirie hier auf die Neuauflage des Duden-Aussprachewörterbuchs gewirkt hat.

11 Vgl. dazu Kleiner (2014, S. 292) und die entsprechenden Anmerkungen unten zu rösten, Ballon und Balkon. 


\subsubsection{Beispiel 1: Aussprache von <ö> in rösten}

Aus etymologischer Sicht liegt mhd. rœsten mit langem Tonsilbenvokal zugrunde, der bei regelmäßiger Weiterentwicklung, wie in den Parallelfällen böse, löten, trösten, im Neuhochdeutschen allgemein als langes/gespanntes [ø:] realisiert wird. Entsprechend verzeichnen die deutschen Aussprachekodizes - bis in jüngste Zeit - entweder nur den Typ ['rø:stn] (z.B. Siebs 1969; Krech et al. 2009) oder dokumentieren immerhin, wie das Duden-Aussprachewörterbuch schon seit der 2. Auflage (Mangold 1974, S. 623), beide Varianten, allerdings mit deutlicher Abstufung: ['rø:stn, auch: 'rœstn].$^{12}$

Die Auswertung der Vorleseaussprache von rösten im Korpus „Deutsch heute“ zeigt, dass die Kodizes in diesem Fall weit davon entfernt waren, den tatsächlichen Sprachgebrauch angemessen zu repräsentieren:

Auf Abbildung 1 stehen schwarze Symbole für den Lauttyp [ø:], weiße für [œ]. Auf den ersten Blick wird deutlich, dass langvokalisches [ø:] nur im Südwesten des deutschen Sprachraums die Mehrheit stellt und insbesondere in der Schweiz die absolut dominierende Variante ist. Außerhalb Baden-Württembergs ist in Deutschland nur im Saarland und im Süden von Rheinland-Pfalz eine hohe Dichte dieser Variante zu erkennen, in Hessen und Sachsen stellt [ø:] immerhin noch mehr als 20\% der Belege, während der Langvokal in Bayern allenfalls noch sporadisch auftritt. Im Norden Deutschlands herrscht dagegen ausschließlich kurzes/ungespanntes [œ]. Ein Vergleich mit der entsprechenden Karte aus dem Ausspracheatlas von Werner König (1989, S. 165) zeigt für die in den 1970er-Jahren in den alten Bundesländern erhobenen Daten noch eine größere Verbreitung von [ø:], das gilt vor allem für Bayern. Auf das Gesamtgebiet der alten Bundesländer gesehen geht der [ø:]-Anteil im 30-Jahres-Vergleich zwischen beiden Korpora von 31\% auf 17\% zurück.

Aufgrund dieser Korpusdaten haben wir die Ausspracheangaben in der Neuauflage entsprechend verändert zu: „rösten 'rœstn, schweiz. vorw., südwestd. auch: 'rø:stn“. Die vormals primäre Variante wurde zu einer nur mehr regional verbreiteten abgestuft, die vormals sekundäre Variante wurde zur unmarkierten Hauptvariante.

12 Die aus den älteren Aussprachekodizes angeführten Formen sind zum Zweck der besseren Vergleichbarkeit typisiert, also nicht zeichengetreu zitiert. 


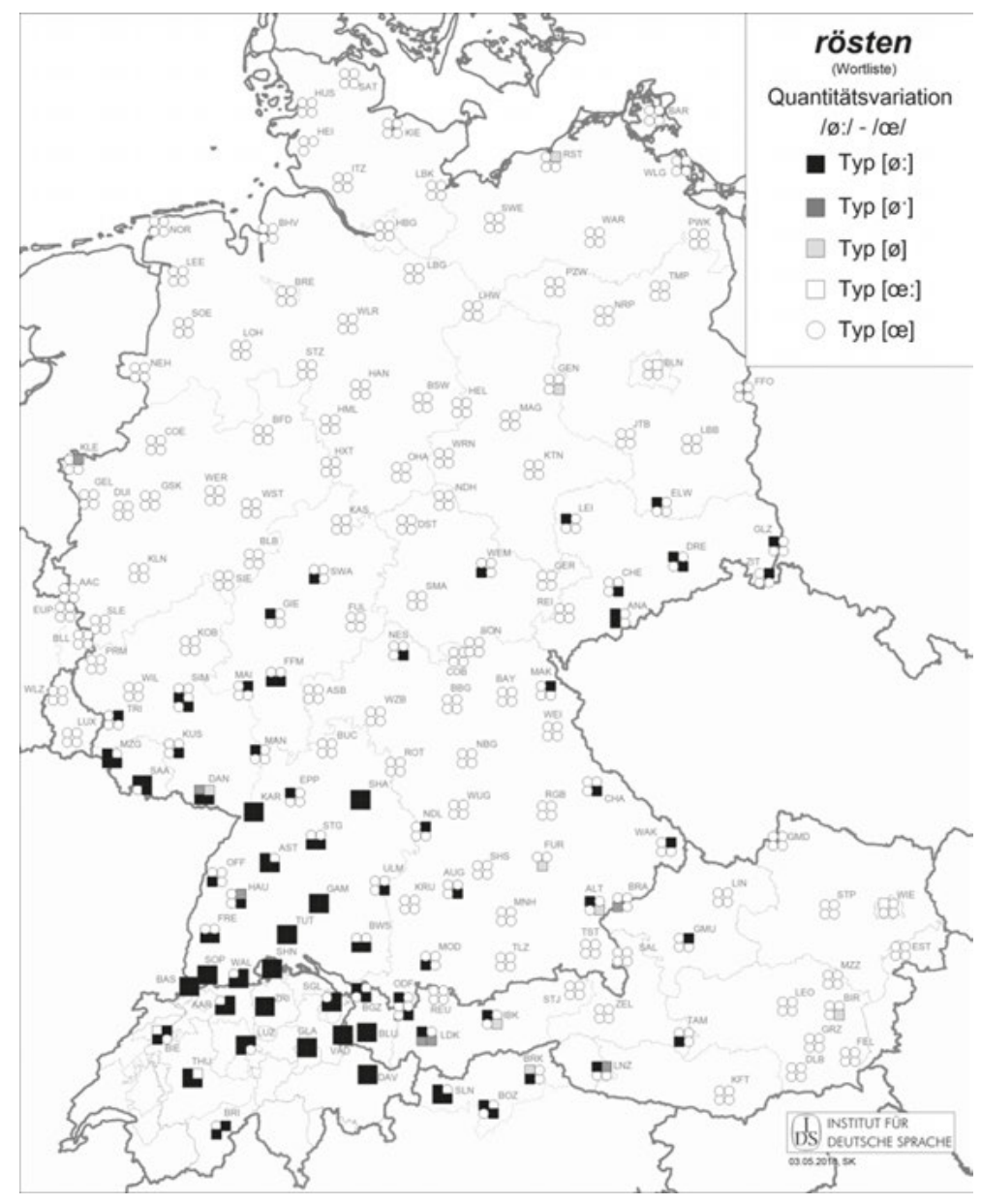

Abb. 1: Aussprachevarianten für <ö〉 in rösten

\subsubsection{Beispiel 2: Aussprache von -<on> in Ballon und Balkon}

Ballon und Balkon gehen zwar letztlich auf italienischen Ursprung zurück, sind aber (nach Pfeifer 1993, S. 91-92) aus dem Französischen ins Deutsche gekommen und auf jeden Fall lautlich davon beeinflusst, was Variation bei der Realisierung der Endung zur Folge hat. Diese Variation ist im Duden-Aussprachewörterbuch bereits seit der 2. Auflage (Mangold 1974) im Prinzip schon abgebildet: Ballon [ba'lõ:, auch: ba'lon, ba'lo:n], parallel dazu Balkon [bal'kõ:, auch: bal'kə习, bal'ko:n]. In anderen Aussprachekodizes fehlt mindestens jeweils eine der eingedeutschten 
Varianten. Siebs (1969, S. 195) verzeichnet bei Ballon nur [ba'lã], aber bei Balkon [bal'kz̃] und [bal'ko:n], Krech et al. (2009, S. 350) buchen parallel [bal'วn, bal'õ:] und [bal'kəy, bal'kõ:].

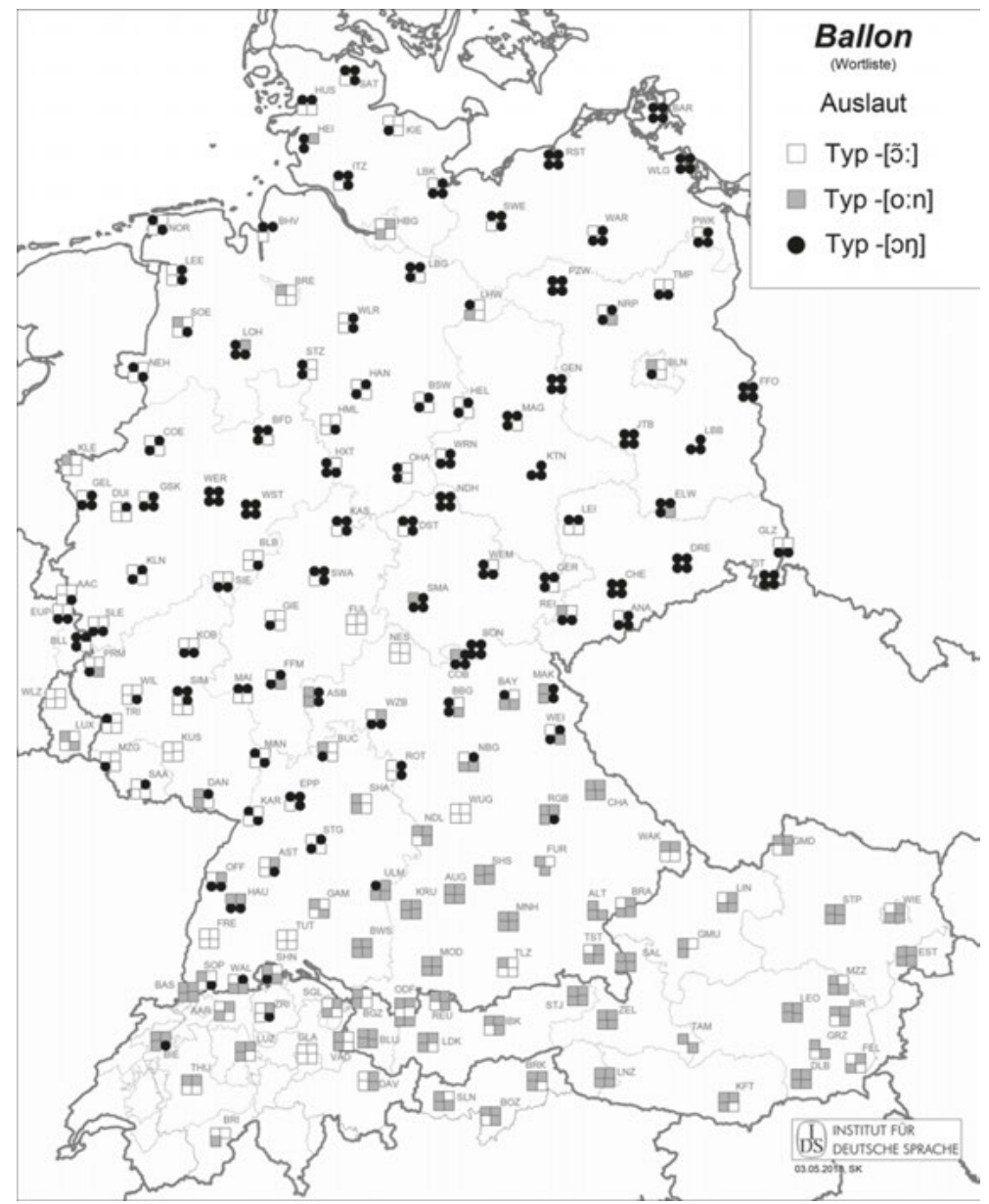

Abb. 2: Realisierung des Auslauts von Ballon 


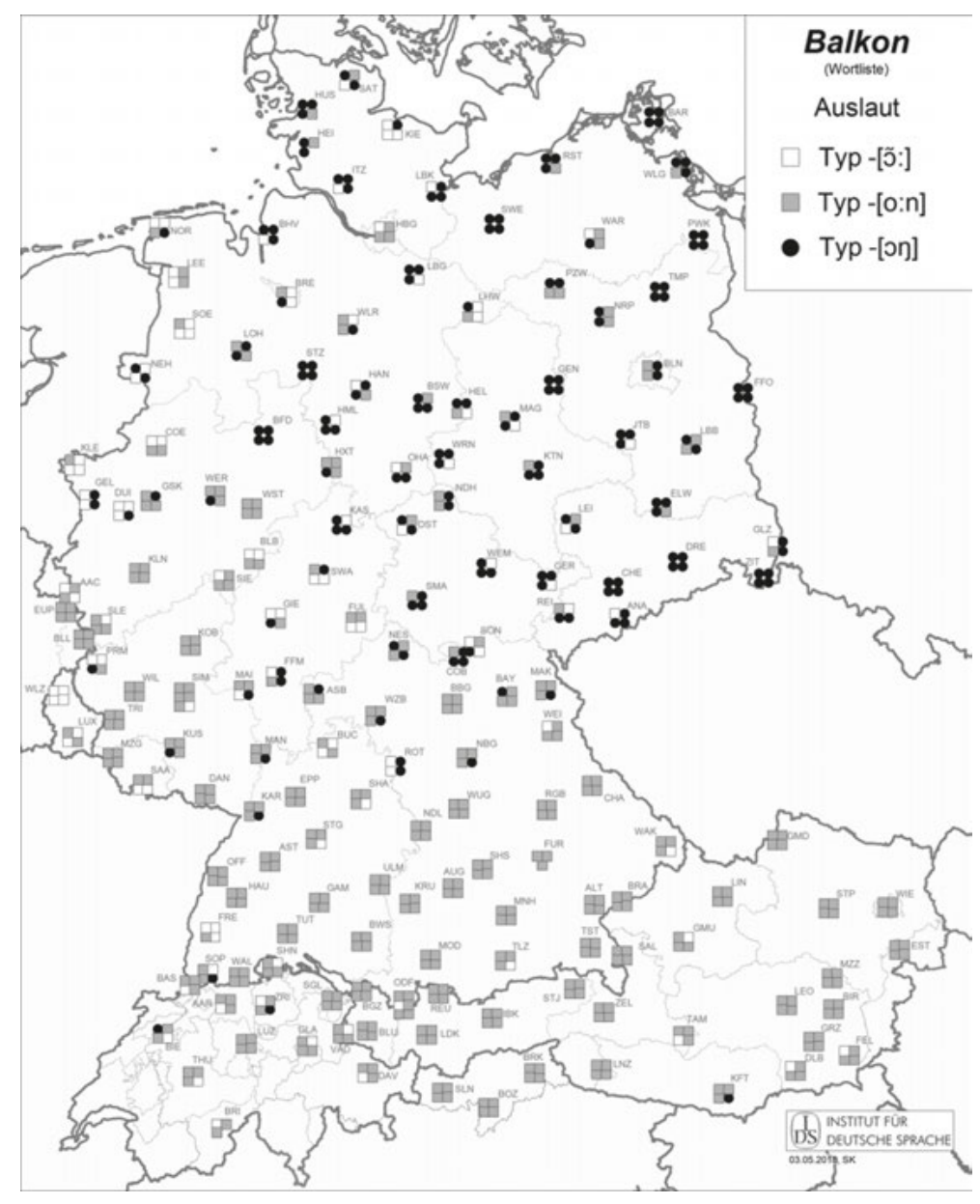

Abb. 3: Realisierung des Auslauts von Balkon

In unseren Korpusdaten (vgl. Abb. 2 und 3) zeigt sich eine klare Raumbildung der beiden eingedeutschten Varianten: In Nord- und Mitteldeutschland dominiert bei Ballon die Aussprache mit Velarnasal und in der Regel kurzem, offenem $o$-Vokal, d.h. der Typ [ba'lon] (symbolisiert durch schwarze Kreise); im Süden (besonders im Südosten) Deutschlands sowie in Österreich und der Schweiz wird hingegen mehrheitlich die Variante mit Alveolarnasal und langem, geschlossenem $o$-Vokal verwendet, d.h. der Typ [ba'lo:n] (symbolisiert durch graue Quadrate). Eine grundsätzlich ähnliche Nord-Süd-Verteilung der Varianten zeigt sich auch bei Balkon, 
allerdings ist hier der [o:n]-Typus wesentlich weiter verbreitet und erstreckt sich auch über den ganzen westlichen mitteldeutschen Raum und streut sogar über fast ganz Norddeutschland. Die an der französischen Aussprache orientierten Realisierungen ohne Nasalkonsonant bei gleichzeitig mehr oder weniger stark ausgeprägter Nasalierung des Vokals vom Typ [ba'lõ:], [bal'k̃̃:] bilden allenfalls bei Ballon, wo sie generell häufiger auftreten (34\%) als bei Balkon (16\%), eine gewisse Konzentration im Westmitteldeutschen, streuen ansonsten aber über das ganze Untersuchungsgebiet. Auf einer hier nicht gezeigten $\mathrm{Karte}^{13}$ ist außerdem dokumentiert, dass der Wortakzent von Balkon in der Schweiz mehrheitlich auf der ersten Silbe liegt. All diese Ergebnisse der Korpusauswertung haben in der Neuauflage zu entsprechend angepassten Wortartikeln geführt: „Ballon ba'lın, ba'lõ:, österr./schweiz. vorw., südd. oft: ba'lo:n“, „Balkon bal'ko:n, bal'kว̃: bes. nordd./ ostd. oft: bal'kon, schweiz. oft: 'balko:n“"

\subsection{Weitere neue Elemente: Infokästen zur Aussprachevariation und mit Umfrageergebnissen}

Abschließend sollen die in der 7. Auflage als neues Informationsvermittlungselement eingeführten Kastenartikel vorgestellt werden, die in anderen Dudenbänden längst Standard sind. Diese ein- oder mehrspaltig angelegten, von minimal knapp zehn Zeilen bis zu eine ganze Seite umfassenden, farblich abgesetzten Text- bzw. Infoblöcke werden im Duden-Aussprachewörterbuch zu zwei verschiedenen Zwecken eingesetzt: Sie geben entweder durch textliche Erläuterungen Zusatzinformationen zur Aussprache(-variation) einzelner Stichwörter oder ganzer, durch grafische oder phonologische Gemeinsamkeiten verbundener Lemmagruppen, oder es werden in ihnen die Ergebnisse einer Online-Umfrage zur Präferenz von Aussprachevarianten gezeigt.

Beispielhaft für die insgesamt 81 enthaltenen Kästen mit Informationen zu Aussprachevarianten sind in Abbildung 4 diejenigen zur Aussprache des Buchstabens $<\mathrm{y}>$ in poly- sowie zum Wortakzentsitz in ausgezeichnet abgebildet.

13 Online publiziert unter http://prowiki.ids-mannheim.de/bin/view/AADG/BudgetAkzent (Stand: 03.08.2018). 


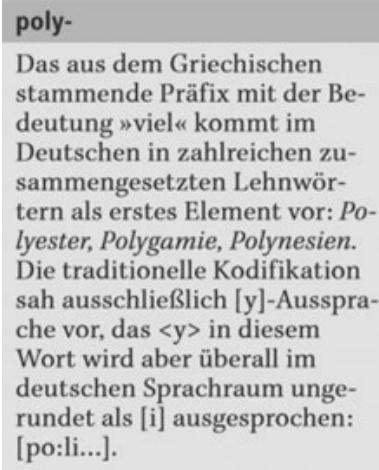

ausgezeichnet
Bei ausgezeichnet wirkt der
Wortakzent häufig bedeu-
tungsunterscheidend: Das Par-
tizip zu auszeichnen (Der Preis
war nicht ausgezeichnet.) wird
immer auf der ersten Silbe be-
tont: ['ausgatsalçnət]. In der
Bedeutung mexzellent, hervor-
ragend « wird dagegen in prädi-
kativer bzw. adverbieller Ver-
wendung meistens auf der
Stammsilbe betont:
[aussg'tsąçnt] (Das Essen
war ausgezeichnet!). In attribu-
tiver Verwendung (Das war ein
ausgezeichnetes Essen!) sind
jedoch auch in dieser Bedeu-
tung beide Akzentuierungs-
muster gebräuchlich.

Abb. 4: Infokästen zur Aussprache von poly- und ausgezeichnet

Im Rahmen dieser Neuauflage wurde außerdem eine Online-Umfrage zur Akzep$\operatorname{tanz}$ von Aussprachevarianten durchgeführt, aus der ausgewählte relevante Ergebnisse im Wörterbuchteil publiziert werden. Die Umfrage bot insbesondere die Möglichkeit, auch zu solchen Wörtern empiriegestützte Angaben zu machen, die in den verfügbaren Sprachdaten nicht oder nicht ausreichend belegt sind. Sie zielte besonders auf die drei Variationsbereiche Wortakzent (Massaker), Vokalquantität (Omega) und die Aussprache von Lehnwörtern (Trolley). Die grundsätzliche Aufgabe der UmfrageteilnehmerInnen bestand darin, den Grad der Angemessenheit der Ausspracheformen in formellen Sprechsituationen anhand einer fünfstufigen Bewertungsskala (Likert-Skala) einzuschätzen. Die TeilnehmerInnen wurden aufgefordert, ihre Angemessenheitsurteile unabhängig vom eigenen Sprachgebrauch zu fällen. Die Skalen zu den einzelnen Varianten bestanden jeweils aus den beiden positiven Bewertungsoptionen ,voll angemessen“ und „weitgehend angemessen“ und den negativen Entsprechungen ,ganz unangemessen“ und ,weitgehend unangemessen“ sowie dazwischenliegend aus der Möglichkeit, die Varianten als „neutral“ zu bewerten. Bei der Präsentation der Umfrageergebnisse sind die beiden zustimmenden und ablehnenden Bewertungskategorien jeweils zu einer zusammengefasst worden (als „+“ bzw. ,-“" bezeichnet), sodass immer drei Prozentwerte pro Variante angegeben werden. Die Umfrageergebnisse haben an einigen Stellen zur Modifikation des Wörterbucheintrags geführt. Bei ihrer Interpretation gilt es allerdings zu beachten, dass es sich dabei primär um Angemessenheitsurteile handelt, also um die Intuitionen der Befragten, die nicht mit empirischen Befunden aus Sprachaufnahmen als Ausschnitten der Sprechrealität identisch sein müssen. 
Bis zum Zeitpunkt der Auswertung für die Neuauflage hatten knapp über 1.000 Personen an der Umfrage teilgenommen. Um die Angemessenheitsurteile sprachräumlich auszubalancieren, wurden aus der Gesamtmenge der befragten Personen Stichproben genommen, die sich an den deutschen Bundesländern bzw. im Fall von Österreich und der Schweiz an den nationalen Einheiten orientierten, in denen die TeilnehmerInnen den größten Teil der ersten 16 Jahre ihres Lebens verbracht haben. In die Auswertung einbezogen wurden letztlich die Angemessenheitsurteile von insgesamt 573 Personen. ${ }^{14}$

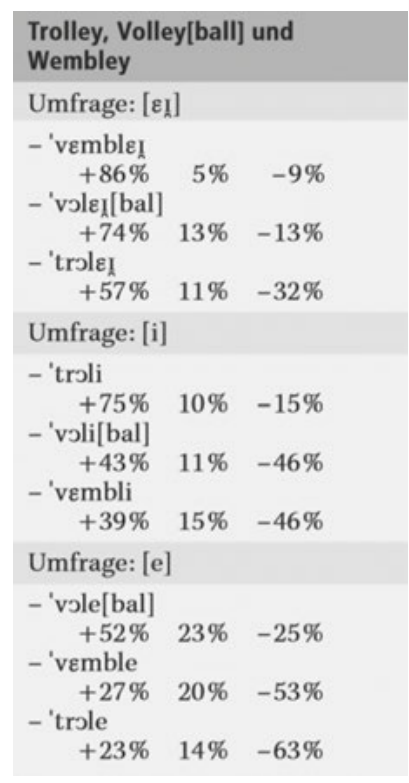

Abb. 5: Infokasten mit Umfrageergebnissen zur Aussprache von <-ey> in Trolley, Volley(ball) und Wembley

Als Beispiel für einen Infokasten mit Umfrageergebnissen wird in Abbildung 5 eines der ausführlichsten Exemplare gezeigt, das Informationen zu gleich drei Wörtern im Vergleich enthält (üblicherweise wird nur ein Wort pro Kasten thematisiert). Hier geht es um die Aussprache der Endung <ey> in den aus dem Englischen stammenden Wörtern Trolley, Volley(ball) und Wembley. In der Umfrage

14 Der Wortlaut dieser Passage wurde in wesentlichen Teilen vom entsprechenden Abschnitt aus der 7. Auflage (S. 15-16) übernommen. 
wurden die drei Varianten [e], [i] und [عI] zur Auswahl angeboten. ${ }^{15}$ Es ist zu erkennen, dass sich die drei Wörter recht unterschiedlich verhalten: Während bei Wembley und Volley (ball) die an der Schrift orientierte [عI]-Aussprache mit Abstand am angemessensten bewertet wird, wird bei Trolley [i] präferiert. Bei Volley(ball) kommt daneben auch die Variante [e] auf mehr als 50\% Angemessenheit. (Vermutlich dürften die Zahlen bei (Eis-)Hockey ähnlich ausfallen.) Diese hohen Angemessenheitswerte sind ohne Zweifel auch ein Reflex des tatsächlichen Gebrauchs, weshalb es überrascht, dass für die Aussprache von <ey> bisher weder [عI] noch [e] als Varianten in Aussprachekodizes verzeichnet waren.

\section{Nicht durchgeführte Änderungen/Desiderate}

Aufgrund der zahlreichen Neuerungen und Aktualisierungen führt die 7. Auflage des Duden-Aussprachewörterbuchs in der Titelei das Etikett „komplett überarbeitet“ sicherlich nicht zu unrecht. Es gibt allerdings Punkte, deren Umsetzung einerseits in der Kürze der zur Überarbeitung zur Verfügung stehenden Zeit, andererseits aus strukturellen Gründen nicht möglich war. So haben wir beispielsweise in einigen Fällen darauf verzichtet, sehr häufige, nicht wortspezifische (und damit vorhersagbare) Varianten bei jedem in Frage kommenden Stichwort abzubilden. Paradebeispiel hierfür ist die Variation zwischen Velarplosiv [k] und Frikativ [ç] in nebentoniger Silbe bei -<ig>-Schreibung. Bei insgesamt ca. 3.000 Stichwörtern, die nebentoniges <ig > enthalten, wären - abgesehen von dem erheblichen Zeitaufwand für die Artikelkorrektur - durch die zusätzlichen Zeilenumbrüche ca. zehn weitere Druckseiten nötig gewesen. Auch bei der allgegenwärtigen Variation von langem / $\varepsilon$ :/ (erzählen, Käse usw.) wurde, wie bisher, nur [ع:] verzeichnet und nicht die besonders in Mittelost- und Norddeutschland sowie in Österreich allgemein gebräuchliche [e:]-Variante. Und bei einer konsequenten Umsetzung der regionalen Variation hätte bei allen mit /z/ + Vokal anlautenden Wörtern die im Süden

15 Die ganze Umfrage wurde nach Phänomengruppen gegliedert, die jeweils blockweise abgefragt wurden; den jeweiligen Blöcken war ein Erläuterungstext vorangestellt. Da es sich um eine Umfrage unter Laien handelte, wurde versucht, die lautlichen Varianten möglichst laienverständlich zu präsentieren. So wurde bei Fragen zum Wortakzent die jeweils betonte Silbe durch Großschreibung hervorgehoben (z.B. KAkadu vs. KakaDU), bei Vokalquantitätsvariation wurde ein nachgestellter Doppelpunkt zur Markierung von Länge und ein übergestellter Bogen zur Markierung von Kürze eingesetzt, und bei segmenteller Variation wurde versucht, die Lautschrift an übliche orthografische Konventionen anzulehnen (z.B. ej für die Variante mit englischem Diphthong, die hier im Text in IPA mit [عI] wiedergegeben wird). 
des deutschen Sprachraums, besonders in Österreich und der Schweiz, allgemein übliche Normalaussprache als [s-] abgebildet werden müssen.

Ebenfalls aus Zeit- und Platzgründen haben wir in der Regel darauf verzichtet, regionale Varianten bei Ableitungen und Zusammensetzungen anzugeben, sie sind nur bei der Ableitungsbasis bzw. dem Grundwort dargestellt. Entsprechend fehlt bei den insgesamt 20 verzeichneten Erweiterungen von Chemie (Chemigraf, Chemikalie, chemisch, Petrochemie usw.) die bei diesem Lemma insgesamt vier Zeilen umfassende Darstellung der Variation des Anlautkonsonanten v.a. zwischen [ç], [f] und [k].

Problematisch an diesen nicht umgesetzten Änderungen ist, dass sie mit der üblichen Praxis der Wörterbuchnutzung nur schlecht kompatibel sind: Wenn man ein bestimmtes Wort nachschlägt, um darüber Informationen, in unserem Fall zu dessen Aussprache(varianten), zu bekommen, sollten diese einerseits möglichst vollständig beim jeweiligen Stichwort aufgeführt sein und andererseits optimalerweise noch einen Verweis auf Stellen enthalten, wo $\mathrm{zu}$ diesen Varianten weitere Informationen $\mathrm{zu}$ finden sind (z.B. zusammenfassende Regelformulierungen im Einleitungsteil oder in Kastenartikeln). Die Varianten in diesem Sinne noch stärker als bisher in den einzelnen Wortartikeln abzubilden, wird für eine zukünftige 8. Auflage eine hohe Priorität haben.

Ganz zum Schluss soll allerdings noch ein weiteres Desiderat angesprochen werden, das insbesondere für ein Aussprachewörterbuch von höchster Relevanz ist, sich aber in der klassischen Publikationsform als gedrucktes Buch nur über Umwege realisieren lässt: Die Aussprache nicht nur gedruckt in Lautschrift abzubilden, sondern auch möglichst für die gesamte Stichwortliste vertont bereitzustellen. Dies ist bei der 7. Auflage erst für ca. 13.000 Stichwörter der Fall, die man sich nach dem Download der Software „Duden-Bibliothek 6“16 anhören kann. Der Bestand deckt sich dabei mit dem, der derzeit im Webportal „Duden online“17 zur Verfügung steht. Allerdings wird vom Dudenverlag eine Vertonung des kompletten über „Duden online“ zugänglichen Bestands (über 200.000 Stichwörter) geplant, von der dann letztlich auch das Duden-Aussprachewörterbuch profitieren wird. ${ }^{18}$

16 Vgl. https://www.duden.de/Shop/Das-Ausspracheworterbuch-1 (Stand: 2.8.2018).

17 Verfügbar unter der Webadresse https://www.duden.de/woerterbuch (Stand: 2.8.2018).

18 Dem „Longman pronunciation dictionary“ (Wells 2008) liegt eine CD mit einer Datenbank bei, auf der alle Inhalte des Buchs, ein Aussprachetrainer und darüber hinaus zu allen Stichwörtern Vertonungen in britischem und, bei Ausspracheunterschieden, in amerikanischem Englisch verfügbar sind. 


\section{Literatur}

Ammon, Ulrich et al. (2004): Variantenwörterbuch des Deutschen. Die Standardsprache in Österreich, der Schweiz und Deutschland sowie in Liechtenstein, Luxemburg, Ostbelgien und Südtirol. Berlin/New York.

Bremer, Otto (1918): Deutsche Lautlehre. Leipzig.

Brinkmann, Caren et al. (2008): German today: An areally extensive corpus of spoken standard German. In: Proceedings of the 6th International Conference on Language Resources and Evaluation (LREC 2008), Marrakech, Morocco. Marrakesch, S. 3185-3191.

Elspaß, Stephan/Möller, Robert (2003 ff.): Atlas zur deutschen Alltagssprache (AdA). Internet: www.atlas-alltagssprache.de (Stand: 15.8.2018).

Haas, Walter/Hove, Ingrid (2009): Die Standardaussprache in der deutschsprachigen Schweiz. In: Krech et al., S. 259-277.

Kleiner, Stefan (2008): Rezension von: Rudolf Muhr: Österreichisches Aussprachewörterbuch. Österreichische Aussprachedatenbank. In: Zeitschrift für Dialektologie und Linguistik 75, 3, S. 369-373.

Kleiner, Stefan (2014): Die Kodifikation der deutschen Standardaussprache im Spiegel der faktischen Variabilität des Gebrauchsstandards. In: Plewnia, Albrecht/Witt, Andreas (Hg.): Sprachverfall? Dynamik - Wandel - Variation. (= Jahrbuch des Instituts für Deutsche Sprache 2013). Berlin/Boston, S. 273-298.

Kleiner, Stefan (2015): „Deutsch heute“ und der Atlas zur Aussprache des deutschen Gebrauchsstandards. In: Kehrein, Roland/Lameli, Alfred/Rabanus, Stefan (Hg.): Regionale Variation des Deutschen. Projekte und Perspektiven. Berlin/Boston, S. 489-518.

Kleiner, Stefan/Knöbl, Ralf/Mangold, Max (†) (2015): Duden. Bd. 6: Das Aussprachewörterbuch. 7., kompl. überarb. u. akt. Aufl. Berlin.

Kohler, Klaus (1995): Einführung in die Phonetik des Deutschen. 2., neubearb. Aufl. (= Grundlagen der Germanistik 20). Berlin.

König, Werner (1989): Atlas zur Aussprache des Schriftdeutschen in der Bundesrepublik Deutschland. 2 Bde. Ismaning.

Krech, Eva-Maria et al. (2009): Deutsches Aussprachewörterbuch. Mit Beiträgen von Walter Haas, Ingrid Hove, Peter Wiesinger. Berlin u.a. [Unter Mitarbeit von Ines Bose, Uwe Hollmach, Baldur Neuber].

Krech, Hans et al. (Hg.) (1964): Wörterbuch der deutschen Aussprache. Leipzig.

Mangold, Max (1962): Der große Duden. Bd. 6: Aussprachewörterbuch. Mannheim u.a.

Mangold, Max (1974): Der große Duden. Bd. 6: Aussprachewörterbuch. Wörterbuch der deutschen Standardaussprache. 2., völlig neu bearb. u. erw. Aufl. Mannheim u.a.

Mangold, Max (2005): Duden. Bd. 6: Das Aussprachewörterbuch. 6., überarb. u. aktual. Aufl. Mannheim u.a.

Muhr, Rudolf (2008): Österreichisches Aussprachewörterbuch. Österreichische Aussprachedatenbank. Berlin/Frankfurt u.a.

Pfeifer, Wolfgang (1993): Etymologisches Wörterbuch des Deutschen. 2. Aufl. Berlin.

Schmidt, Jürgen E./Herrgen, Joachim (2011): Sprachdynamik. Eine Einführung in die moderne Regionalsprachenforschung. (= Grundlagen der Germanistik 49). Berlin.

Siebs, Theodor (Hg.) (1898): Deutsche Bühnenaussprache. Berlin u.a.

Siebs, Theodor (1969): Deutsche Aussprache. Reine und gemäßigte Hochlautung mit Aussprachewörterbuch. Hrsg. v. Helmut de Boor, Hugo Moser und Christian Winkler. Berlin. 
Wells, John C. (1990): Longman pronunciation dictionary. Harlow.

Wells, John C. (2008): Longman pronunciation dictionary. 3. Aufl. Harlow.

Wiesinger, Peter (2009a): Rezension von Rudolf Muhr: Österreichisches Aussprachewörterbuch. Österreichische Aussprachedatenbank. In: Beiträge zur Namenforschung 44, 2, S. $243-250$.

Wiesinger, Peter (2009b): Die Standardaussprache in Österreich. In: Krech et al., S. 229-258. 